\title{
Muscle hypertrophy and muscle strength: dependent or independent variables? A provocative review
}

\author{
Carlo Reggiani (1,2), Stefano Schiaffino (3)
}

(1) Department of Biomedical Sciences of the University of Padova, Padova, Italy; (2) Science and Research Centre Koper, Institute for Kinesiology Research, Koper, Slovenia; (3) VIMM Venetian Institute of Molecular Medicine, Padova, Italy

This article is distributed under the terms of the Creative Commons Attribution Noncommercial License (CC BY-NC 4.0) which permits any noncommercial use, distribution, and reproduction in any medium, provided the original author(s) and source are credited.

\begin{abstract}
The question whether the muscle hypertrophy induced by resistance training, hormone administration or genetic manipulation is accompanied by a proportional increase in muscle strength is still open. This review summarizes and analyses data obtained in human and rodent muscles in studies that have monitored in parallel changes in muscle size and changes in muscle force, measured in isometric contractions in vivo, in isolated muscles ex vivo (in rodents) and in single muscle fibers. Although a general positive relation exists among the two variables, a number of studies show a clear dissociation with increase of muscle size with no change or even decrease in strength and, vice versa, increase in strength without increase in size. The possible mechanisms of such dissociation, which involves neural motor control and/or cellular and molecular adaptations of muscle fibers, are briefly discussed.
\end{abstract}

Key Words: skeletal muscle, hypertrophy, resistance training, contractile force.

Eur J Transl Myol 2020; 30 (3): 9311. doi: 10.4081/ejtm.2020.9311

In the competitive sport world, several disciplines involve high level of muscle hypertrophy, as for example weight lifting and throwing sports (shot put, discus throw and hammer throw) among the field and track events. Actually, all these sports require a specific training, aimed at increasing muscle performance in terms of force and explosive power and based on resistance exercise, i.e., repeated muscle contractions against a resistance or a weight, and this type of training produces a significant muscle hypertrophy. At first sight, similar training protocols are adopted in a parallel and independent world, which is represented by bodybuilding. Athletes who train for bodybuilding develop highly hypertrophic muscles with specialized protocols of resistance training. If we compare by eye a body builder and a weight lifter we can detect differences and similarities in their body structure. Both have large muscle mass in limbs and trunk, which in athletes trained for throwing sports and power lifting are often covered by subcutaneous fat, while in body builders the combination of bulking and cutting phases remove all unnecessary fat to make muscles well visible. ${ }^{1}$ Some degree of asymmetry between left and right limbs is often detectable in trained athletes of throwing sports but never in body builders. If we then determine the contractile performance, for example by measuring the maximal voluntary contraction (MVC), we will likely discover that trained athletes perform better than body builders. ${ }^{2}$ Thus, if we ask a coach about the training protocols, which are followed by a body builder and a power lifter, the answer will be that they are very different. In both cases, the athletes will perform repeated sets of contractions against resistance, mostly given by weights or elastic bands, but the distribution of volume (number of sessions per week, or sets in each session or repetitions in each set), intensity (load or resistance), timing (intervals between sets or between exercise sessions) will be adapted to the final goal and the same holds for the nutritional support. This implies that different pathways of training have to be followed if the goal is to maximize hypertrophy or to maximize performance. ${ }^{3}$ The same question, i.e., the relation between increasing muscle mass and improving muscle performance, has been asked in the world of muscle biology and physiology for many years. The pioneering work of Rasch, published in $1955,{ }^{4}$ asked openly whether a "relationship exists between hypertrophy and increase in strength" starting from the critical statement that "it seems unlikely that any simple, direct correlation exists". A key advancement on this issue was the identification of two phases in the resistance training, a first phase where the increase in strength was neural in origin and a second phase where 

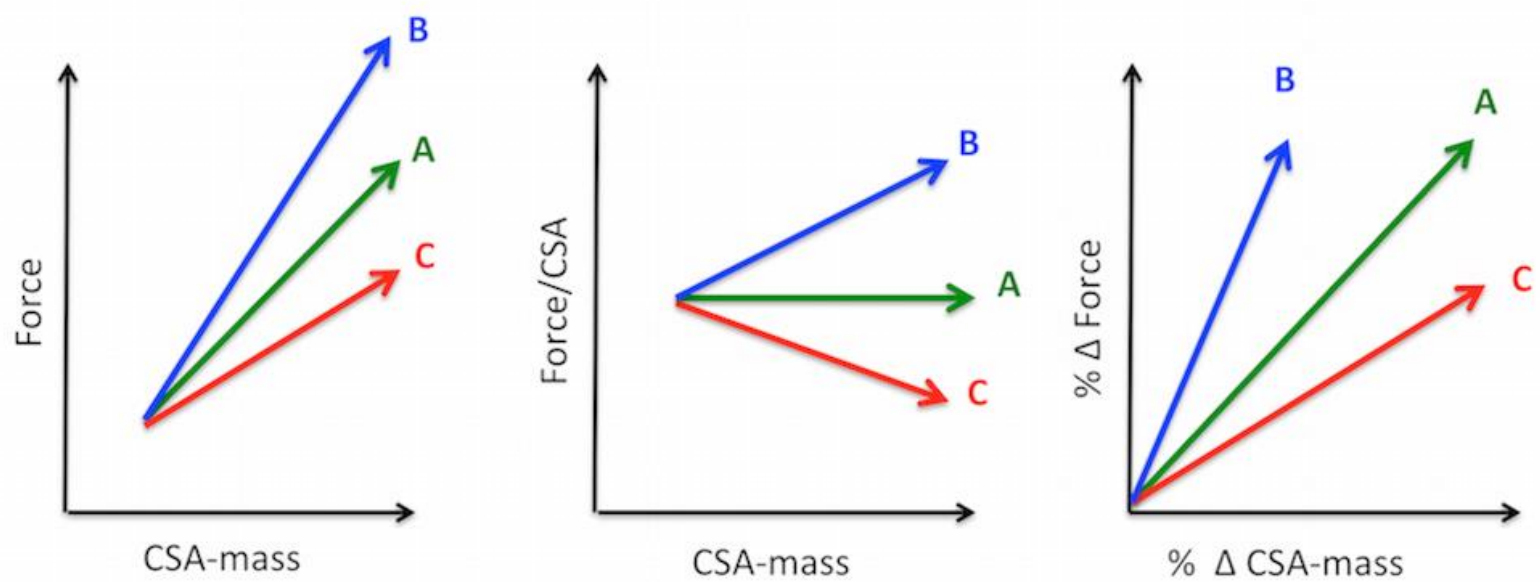

Fig 1. Cartoon showing three alternative relations between increase in muscle size, measured as mass or Cross Sectional Area (CSA-mass) and increase in muscle strength. Force can be normalized to CSA, specific force or tension. Both size and strength can be expressed as \% change (\% $\Delta)$ of initial values. Alternative A: force and size increase in proportion, their ratio remains constant, Alternative B: force increases more than size, their ratio increases, Alternative $C$ : force increases less than size, their ratio decreases.

muscle hypertrophy becomes the dominant factor in strength gain (Moritani and De Vries 1979). ${ }^{5}$ More recently, however, the relation between hypertrophy and increased contractile strength has been critically revisited by several authors. ${ }^{6-9}$ A debated issue is whether the hypertrophy is required to increase performance, and an even more general question is whether the increase in muscle mass is always associated with an increased contractile performance. This latter question is summarized in the cartoon in Figure 1, where three alternative responses to resistance training or to hormone administration are depicted: i) strength increases in proportion to muscle size (A), ii) strength increases more than size (B) and iii) strength does not increase or increases less than muscle size $(\mathrm{C})$. The present short review aims to discuss under this perspective some of the available data from experimental works done in humans and in animal models.
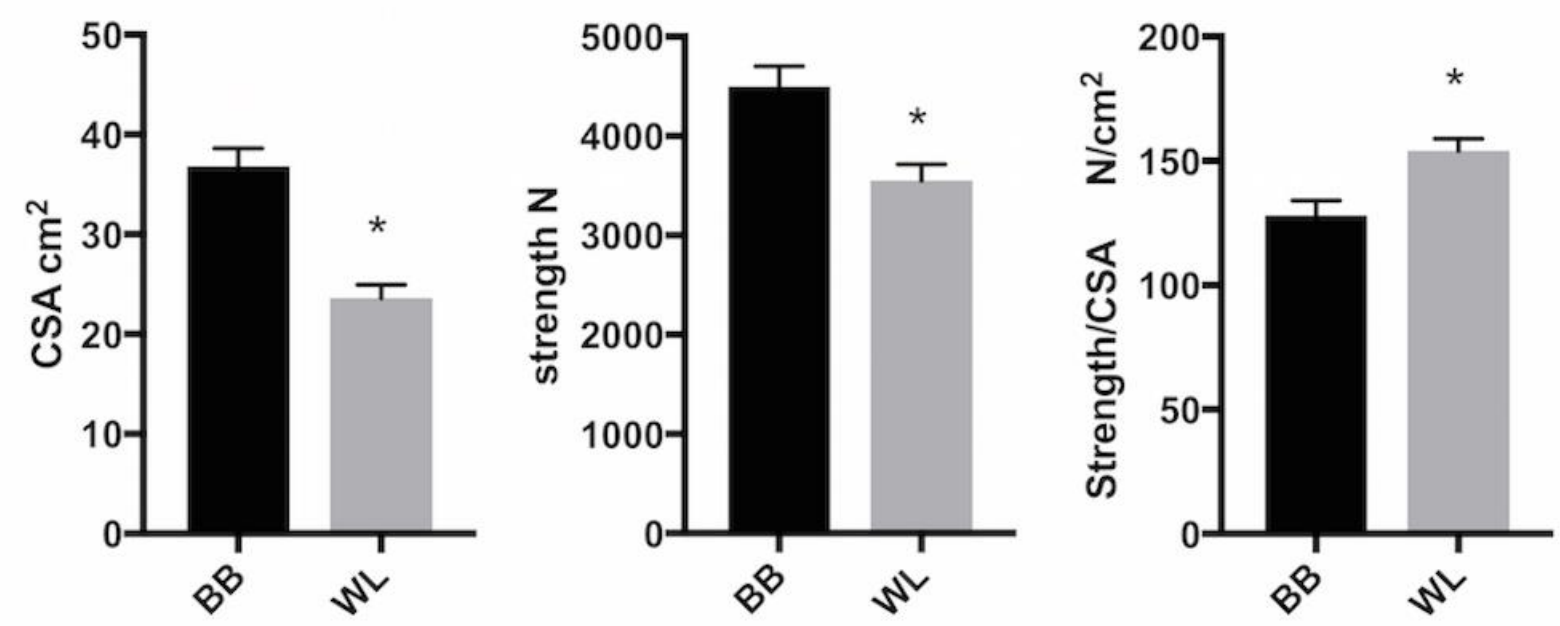

Fig 2. Average values of triceps size and triceps isometric strength in high level body builders and weightlifters. Size is expressed as triceps cross sectional area (CSA) determined with ultrasonography. Strength of arm extensor is measured at the wrist with elbow at $80^{\circ}$ and is then normalized to CSA. Body builders $n=32$, weightlifters $n=20$, means and standard errors, $* p<0.05$. Original figure from data reported in Ikegawa et al. $2008^{2}$ 


\section{Human resistance training: gain in mass and gain in strength}

The number of skeletal muscle fibers is by and large fixed from the first year of life and a skeletal muscle hypertrophy is the physiological process, which allows transversal and longitudinal growth of muscles during childhood and puberty. This occurs mainly under hormonal (steroids and GH/IGF-1) control and with the mechanical stress applied by bone longitudinal growth and by increasing loads in relation to body weight growth and physical activity. After the end of maturation, skeletal muscle hypertrophy can be achieved in the frame of training protocols aimed to increase muscle performance or muscle size.

As discussed above, two main models of skeletal muscle hypertrophy in humans should be considered, both based on resistance training. The first model is based on training protocols aimed to increase power or explosive strength, while the second is based on training protocols aimed just to increase muscle mass, so called body building. ${ }^{3,10}$ A clear example of differential outcome in terms of hypertrophy, strength and strength normalized to muscle size is reported in Figure 2, which shows how two distinct training protocols, selected by body builders and, respectively, by weight lifters, lead to enhanced muscle size or, respectively, muscle strength. ${ }^{2}$

Several studies have compared the determination of force in vivo in humans with the muscle size, most often on leg extensors (Quadriceps, Vastus Lateralis) or on arm flexors (Biceps). The degree of hypertrophy is determined with magnetic resonance imaging (MRI), computed tomography (CT), dual-energy x-ray absorptiometry (DEXA) and ultrasound echography. The latter technique allows the collection of data on muscle architecture, for example pennation angle, in vivo. Force is measured during MVC in isometric conditions, often with interpolated twitch to assess the level of activation, or during movements using isokinetic dynamometers, sometimes with conflicting results. ${ }^{11,12}$ The most accurate reference for force is given by the physiological crosssectional area (PCSA) of the muscle, that accounts for the change in pennation angle, which invariably accompanies the development of hypertrophy.

The results available leave many doubts about the correlation between force and size. For example, Ahtiainen et al. ${ }^{13}$ and Erskine et al. ${ }^{14}$ found very weak associations between increase in mass and increase in force in large (>200) groups of young healthy people, which included both high responders and low responders to the training protocol. As can be seen in Figure 3 (from Ahtiainen et al. ${ }^{13}$ ), 20-24 weeks of resistance training were followed by an average increase in force by $21 \%$, with an average increase in size by $7 \%$. Several individuals (low responders) were able to improve their lower limb strength without any increase in muscle mass (lower right quadrant), while very few increased their muscle size without functional improvement (upper left quadrant). These recent results are consistent with the weak correlation between force and size reported in the pioneering study by Maughan and coworkers. ${ }^{15} \mathrm{~A}$ negative correlation between strength normalized to cross-sectional area (CSA) and CSA was observed by Alway et al. ${ }^{16}$ in the arm flexors of recreational resistance trained subjects. This latter finding suggests that the relation between strength and CSA is not linear.

A living debate is ongoing as to the impact of the modalities of resistance training on muscle size and strength. As shown by Schoenfeld and coworkers, ${ }^{17}$ high training volume, i.e. high number of repetitions of a given exercise, is instrumental in the increase of muscle mass but not muscle strength. There is evidence, however, of a ceiling or a maximal volume per week above which no further gain in volume can be achieved. ${ }^{18}$. The increase of strength is similar regardless of the exercise volume, as one set training may be similarly effective at increasing muscular strength as three or five sets per exercise, but it is very sensitive to the load (intensity). For this reason high volume training, i.e. more sets at lower load, is preferred by body builders. ${ }^{3,10}$ There are further indications that varying volume, intensity and timing of the exercise lead to different degrees of hypertrophy and possibly also to different quality of hypertrophy. ${ }^{3}$

Among the possible explanations of the lack of a robust correlation between increase in mass and gain in strength is the contribution of neural adaptations, as training implies learning of the relevant motor unit recruitment and firing rate and simultaneous de-activation of antagonist muscles. ${ }^{5,19}$ Some protocols of resistance training might not be suitable to sufficiently develop the neural control on the hypertrophic muscles, while some other protocols might be sufficient to improve neural motor control but not to stimulate hypertrophic growth. Inter-individual differences and adaptations of tendon compliance to training could further dissociate the force generated by the hypertrophic muscles and from that exerted on the bones and measurable with a dynamometer. ${ }^{20}$

The analysis of the correlation between hypertrophic growth and increase of force at the level of single muscle fibers removes any possible interference of the neural control and of the tendon compliance. A number of longitudinal studies on the response of single muscle fibers to resistance training prolonged from a few weeks (12 in most studies) up to 1 year (see for example: Widrick et al. ${ }^{21}$, Pansarasa et al. ${ }^{22}$ ) consistently show that specific tension, i.e. maximal isometric force/cross sectional area, is unchanged or even increased by hypertrophy. Similar results were obtained with a transversal comparison between myofibres from people trained with resistance training for 7 years with those of untrained people. ${ }^{23}$ This indicates parallel or proportional variations of fiber size and fiber contractile performance. In contrast with these results, two studies on isolated hypertrophic fibers of body builders, have shown that in well trained professional body builders increase in size 


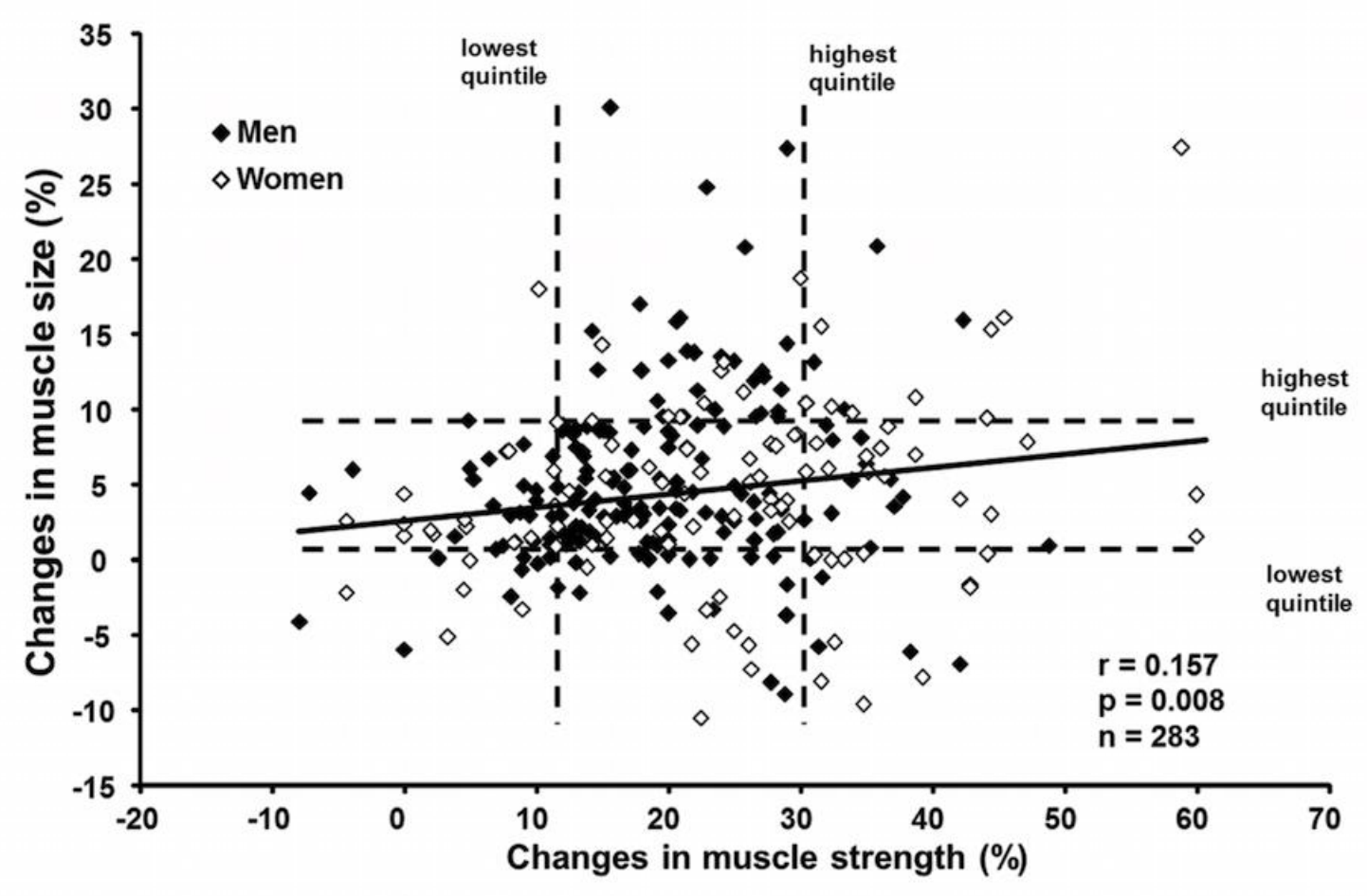

Fig 3. Relation between increase in leg extensor force and increase in quadriceps cross sectional area in a heterogeneous population $(n=283)$ at the end of 20-24 weeks of resistance training. An overall correlation (continuous line) is present ( $r=0.157, p=0.008)$. However, individuals who gained strength and lost muscle mass and individuals who increased their muscle mass without gaining force are easily detectable. Dashed lines allow identification of the lowest and highest quintiles in changes of muscle size and strength. From Ahtiainen et al. 2016 with kind permission of the American Aging Association. ${ }^{13}$

and in force are dissociated, resulting in a lower specific tension for all fiber types, ${ }^{24}$ or only for slow fibers. ${ }^{25}$ Since determination of isometric force in single fibers $e x$ vivo is carried out after sarcolemma permeabilization with a full supply of ATP and complete maximal calcium activation, the discrepancy likely finds a structural basis. The search for such structural basis brings to a debated issue: how much myofibrils and how much sarcoplasmic proteins and organelles contribute to hypertrophic growth or in other terms what is the balance between myofibrillar and sarcoplasmic hypertrophy. Transmission electron microscopy (TEM) studies aimed to quantify the two components have shown that, although myofilament packing is unchanged in fibers from body builders, ${ }^{26}$ the fractional volume occupied by myofibrils is reduced with a corresponding increase in the space occupied by sarcoplasmic components. ${ }^{27,28}$ Accumulation of glycogen in the sarcoplasm of myofibers of body builders has been reported by Mac Dougall et al. ${ }^{29}$ Thus, the protocols adopted by body builders could alter the proportion between the myofibrillar and the sarcoplasmic fraction, organelles included, as discussed by Haun et al. (2019) ${ }^{30}$ and Roberts et al. (2020). ${ }^{9}$ This could, in turn, leads to a reduced development of specific tension.

The discrepancy between myofibrillar and sarcoplasmic components of hypertrophy has been investigated also with the determination of the protein fractional synthesis rate (FSR) of the two components using $\mathrm{D}_{2} \mathrm{O}$ as probe. As shown by Brook et al (2015), ${ }^{31}$ myofibrillar protein synthesis is enhanced mainly in the first weeks of training of leg extensor muscles and then shows a decay in the following weeks. The activation of protein synthesis with resistance training is accompanied by increased phosphorylation of mTORC1 and its substrates, p70S6K1 and S6rp, and in addition to decreased eEF2 phosphorylation. The change in phosphorylation of these proteins tends to fade over time. ${ }^{32}$ A similar approach applied not only to the myofibrillar component but also to the sarcoplasmic component, ${ }^{33}$ shows that the early decline of the myofibrillar FSR is accompanied by a 
trend to an increase in the FSR of the sarcoplasmic component. A recent study on a high volume resistance training, ${ }^{30}$ which is favored by body builders, seems to confirm a decrease in concentration of myofibrillar proteins (actin and myosin) and an increase in the sarcoplasmic component.

Altogether, these observations provide support to the idea that body builder muscle fibers might be less gifted in developing force and power due to a limited accumulation of myofibrillar proteins.

A further interesting protocol to induce hypertrophy in humans is the combination of resistance training and blood flow restriction, indicated with the acronyms BFRRE (Blood Flow Restricted Resistance Exercise) or simply BFR (Blood Flow Restricted). The exercise with relative low load with blood flow restriction is able to produce significant hypertrophy in a relatively short time, definitely more than a training with similar protocol without ischemia. ${ }^{34}$ With sufficiently high frequency of the sessions, the increase in muscle fiber CSA can reach almost $+40 \%$ compared to pre-training values, ${ }^{31}$ with increment in MVC of $+7 \%$ only. This suggests a component of edema in the first period of training, later followed by a "true" hypertrophy. Significant increases in satellite cells and myonuclei accompany the development of the hypertrophy. ${ }^{35}$

Some information is available also on hypertrophy induced by testosterone treatment in humans, in relation to the administration of this compound to sarcopenic elderly or cachectic patients to counteract atrophy and to the use as a doping in athletes and body builders. In a longitudinal study, Fitts et al. $(2015)^{36}$ described parallel increase in fiber diameter and isometric force without any change in maximum shortening velocity in both slow and fast fibers, slow fibers showing a greater responsiveness. A greater sensitivity to androgens of slow fibers in terms of force and size has been also confirmed by Lamboley et al. $(2018)^{37}$ in prostate cancer patients exposed to an anti-testosterone treatment. Specific tension was reduced only in slow fibers and indications of a slow-to-fast fiber type transition were observed, thus confirming a specific response of slow fibers.

\section{Animal Studies}

Animal models of resistance training meet several limitations, as it is difficult to create experimental conditions, which motivate animals to overload their muscles. Climbing a steep ladder to reach a reward, with a load applied on the tail or on the back can be an example. ${ }^{38}$ The ablation of synergist muscles as a model of muscle hypertrophy was first introduced by Goldberg in $1967,{ }^{39}$ and has been often used in rodents, ${ }^{40}$ however it is very far from physiological conditions.

In contrast, genetic manipulation of the signalling pathways which control protein synthesis/degradation and hormone administration can be easily implemented in small rodents. Thus, four main models of muscle hypertrophy have been implemented in rodents.
1. synergist ablation, where compensatory hypertrophy develops in few days after a chronic overload, which is generated by surgical removal of all or part of synergistic muscles. Plantar flexors, and less frequently dorsal flexors of the ankle are the muscles involved in this model

2. simulation of resistance training

3. administration of anabolic hormones or pharmacological compounds

4. genetic manipulation of the signalling pathways controlling muscle growth

The two latter models find equivalent also in other animal species, either for reasons related to commercial interest, for example administration of hormones to cattle or poultry, or for spontaneous genetic mutations, for example the myostatin null mutation in belgian blue cows.

The availability of those animal models allows the investigation of functional counterparts of muscle hypertrophy with experimental approaches not possible in human muscle models, for example the study ex vivo of isolated whole muscles or experimental manipulations by pharmacological or genetic approaches using control animals with identical background. Although a large number of experimental studies has applied the synergist ablation in rodents to study muscle hypertrophy, only a limited number has considered the functional changes which accompany the hypertrophic response and can be summarized as loss of contractile force, prolongation of the time parameters, slight increase in resistance to fatigue and impaired energy metabolism. The depression of contractile force of both isolated plantaris and soleus occurs during the first weeks after excision of the synergist gastrocnemius muscle (3-30 days, ${ }^{41}$ or 14 days $^{42}$ ). In the first week, hypertrophy is accompanied by edema and cell infiltration so that fiber CSA increase is also lower than whole muscle mass increase. The functional contractile deficit persists, as isometric force increases less than CSA even after several months. ${ }^{41}$ In contrast, working on skinned single muscle fibers, Mendias et al. $(2017)^{43}$ showed that specific tension had recovered 28 days after synergist ablation, thus suggesting that alteration outside the fibers, such as accumulation of extracellular matrix (ECM), could be responsible for the lower specific tension of isolated whole muscles. Combining mechanical analysis with expression studies (RNA and proteins) Perez-Schindler et al. $(2013)^{42}$ suggested that the fast-to-slow transition in isoform expression (both in myofibrils and in SR) that is known to accompany the hypertrophy of overloaded plantaris muscle could explain both the reduced contractile force (slow fibers develop lower specific tension than fast fibers) and the prolongation of timing parameters and resistance to fatigue.

The principle of resistance training in animals is similar to that in humans, i.e. a training based on the movement of body segments with application of a progressively increasing load. Various protocols have been designed 
for this purpose, for example inviting rats to climb a vertical ladder carrying a progressive load on the tail (5 days per week, during 8 weeks, ${ }^{44}$ or 4 days per week for 26 weeks ${ }^{45}$ ) to receive a reward. With this protocol, hypertrophy of EDL $(+12 \%)$ and soleus $(+15 \%)$ was not accompanied by any change in specific tension and by a modest improvement in fatigue resistance..$^{45}$

Hypertrophy of rat adductor longus was achieved by Roy et al. (1997) ${ }^{38}$ by training rats to move from quadrupedal to bipedal posture carrying increasing loads in response to a light signal. The hypertrophy (muscle weight/body weight $+16 \%$ ) was not accompanied by any change in force or in time to peak twitch contraction and time to half-relaxation, while a reduction in maximum shortening velocity and a small but significant increase in resistance to fatigue were detected. A novel voluntary weightlifting model, which elicits squat-like activities against adjustable load during feeding has been recently implemented for mouse (Cui and coworkers (2020). ${ }^{46}$ Hypertrophy (CSA +14\%) of hindlimb muscles accompanied by increased contractile performance of plantar flexor muscles was achieved in 8 weeks with a significant improvement in insulin response but without changes in fiber type distribution or energy metabolism. ${ }^{46}$ Two other models of exercise leading to muscle hypertrophy were recently developed in mice: a progressive weighted wheel running protocol, which was reported to increase myofiber size, although muscle function was not examined, ${ }^{47}$ and a high intensity interval training protocol based on treadmill running by progressively increasing the angle of incline and speed of the treadmill. ${ }^{48,49}$ The latter protocol leads to significant muscle hypertrophy with a parallel increase in muscle force which is dependent on satellite cell proliferation and fusion, because it is abolished by the genetic ablation of myomaker, a muscle specific membrane protein essential for myoblast fusion. ${ }^{49}$

Several interesting models of hypertrophy in rodents are based on chronic administration of anabolic hormones or chemical compounds. Androgens and, although less studied estrogens, are endowed with a powerful anabolic action. Some rodent muscles, as levator ani (LA), are specifically responsive to androgen stimulation, thus creating a peculiar sexual dimorphism. ${ }^{50,51}$ All skeletal muscles, however, respond to androgen chronic administration with hypertrophic growth, which is more evident in female and castrated animals. In rat plantar flexor muscles (Gastrocnemius, Soleus, Plantaris) slow fibers were found to be more responsive $(+35-40 \%$ increase in diameter at the end of 12 weeks treatment) than fast fibres. ${ }^{52}$ Chronic treatments in adult male mice showed that fast-twitch muscles (EDL and Tibialis) are more sensitive to androgen withdrawal than slow-twitch muscle (Soleus), but that soleus may be more sensitive to androgen administration. ${ }^{53}$ Isometric force changes in proportion to muscle size, however androgen administration improves fatigue resistance in Soleus muscle.
The hypertrophic growth induced by chronic administration of beta agonist, such as clenbuterol or fenoterol, causes a proportional increase of isometric force and muscle size, ${ }^{54-56}$ or even a greater increase in force than in size leading to higher specific tension. ${ }^{57}$ The hypertrophy induced by beta agonist is also accompanied by a slow-to-fast fiber type shift and more pronounced hypertrophic response in fast fibers. The responses of skeletal muscle fibers to beta agonists are abrogated by ablation of the beta-arrestin gene. ${ }^{57}$

When hypertrophy is induced by genetic modifications of the signalling pathways controlling muscle fiber growth, pronounced dissociations between the ability to develop force and muscle size have been reported. Null mutations of myostatin, spontaneous ${ }^{58}$ or engineered in mice, induce huge hypertrophy, possibly also accompanied by hyperplasia, without corresponding increase in force generation, ${ }^{59}$ thus with a reduction of specific tension. The contractile impairment has been attributed to altered intracellular calcium dynamics. ${ }^{60}$ In contrast short-term (3 wk) activation of the Akt-mTOR pathway via inducible expression of a constitutively active Akt in adult mice was followed by marked hypertrophy $(+50 \%$ of muscle mass) accompanied by increased force generation, both in vivo and ex vivo in intact isolated diaphragm strips, and in single-skinned muscle fibers. ${ }^{61}$ IGF-1 constitutive overexpression under the MCL1f promoter was followed by a pronounced hypertrophy which could reach $+40 \%$ in fast fibers and in fast muscles without significant changes in fiber type distribution. ${ }^{62}$ Determination of isometric tetanic force in EDL, ${ }^{62,63}$ and in FDB (Flexor Digitorum Brevis) muscles ${ }^{64}$ showed that active force increased in proportion to muscle size and, thus, specific tension was unchanged. More detailed study on FDB single fibers of transgenic mice overexpressing IGF1 under MLC1f promoter confirmed that average cross-sectional area and tetanic force almost doubled in single muscle fibers, so that specific force was the same in both preparations. ${ }^{64,65}$

\section{Potential mechanisms for dissociation between hypertrophy and force}

According to the most simple and straightforward way of reasoning, hypertrophy should be accompanied by a proportional increase of active contractile force. If hypertrophy implies build up of more myofibrils and more myosin motors and if each myosin generates the same force, force should increase in close proportion to increase of muscle mass during a maximal activation. However, the brief review of the available literature in humans and animals reported in this paper shows that the situation is much more complex. Three alternative cases are depicted in the cartoon of Figure 1 and only one of them assumes a proportional increase of force and mass (case A). Since there are many conditions leading to case $\mathrm{B}$, force increases more than mass, and even more leading to case $\mathrm{C}$ (force increases less than mass), it is worth discussing how the dissociation between force and 


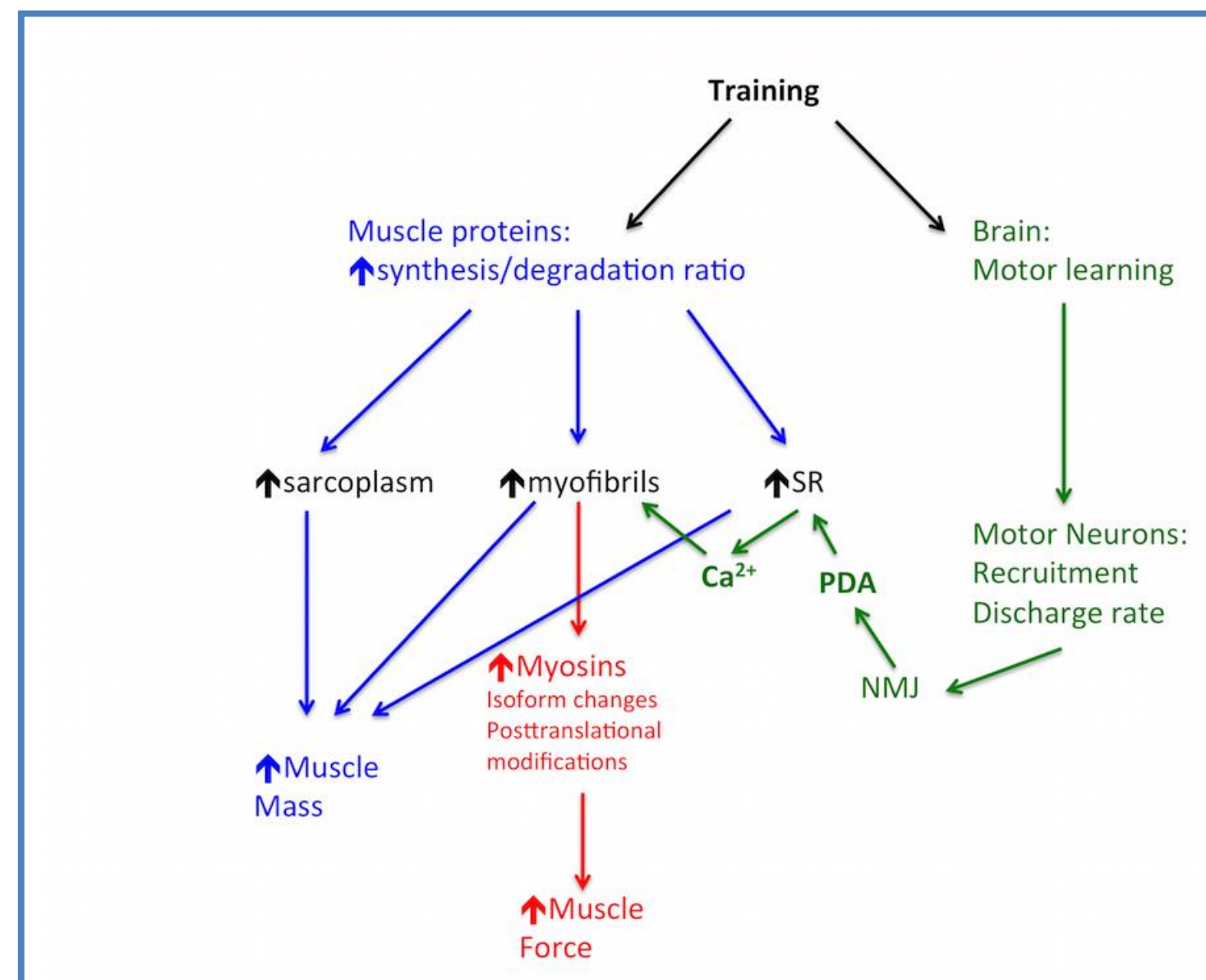

Fig 4. Cartoon showing the most important steps leading to increase in mass and in strength in skeletal muscles. The pathway controlling muscle contractile strength is indicated in red (molecular mechanism of contraction) and green (neural control and intracellular calcium signal), while the factors supporting hypertrophy are indicated in blue.

muscle mass can occurr. Actually, the comparison between slow and fast fibers can be of help for this discussion. Taking as a model the human vastus lateralis, single fibers of similar size develop different force in relation to their type (slow vs fast). The lower tension developed by slow fibers finds its explanation in a lower density of myofibrils related to a larger volume occupied by mitochondria and nuclei, and a lower force generated by individual myosin motors, ${ }^{66}$ which is only partially compensated by a greater fraction of attached myosinactin bridges. ${ }^{66}$

The cartoon in Figure 4 summarizes the main steps leading to increase in mass and, respectively, in isometric strength in skeletal muscles. Although the accumulation of proteins determines an increased abundance of myosin motors, the proportion between sarcoplasmic proteins and myofibrillar proteins could be altered during development of hypertrophy thus producing a higher or lower density of molecular motors. ${ }^{9,30}$ There are several lines of evidence supporting this view, given by TEM, ${ }^{27,28}$ and by determination of glycogen content. ${ }^{29}$ It is important, however, to underline that this evidence only comes from some specific models of hypertrophy. In addition, the functional properties of myosin isoforms might change and affect not only maximum shortening velocity or ATPase activity but also force generation. ${ }^{67}$ The difference in stiffness and in cross bridge kinetics can lead to higher force generation in fast than in slow fibers. ${ }^{66}$ Thus, if hypertrophy is associated with a myosin isoform shift (slow-to-fast or vice versa), also a change in specific tension can be expected. Further variations in myosin properties including force development can be due to post-translational modifications. There are several examples of such modifications, which involve slow myosin in body builder muscles, ${ }^{24}$ fast $2 \mathrm{~A}$ myosin after 12 weeks of resistance training, ${ }^{68}$ and myosins of elderly heart failure patients exposed to resistance training. ${ }^{28}$ Finally, the activation of the thick filament, ${ }^{69,70}$ and thin filament, ${ }^{71}$ might change with resistance training. In particular, it has been shown that intracellular calcium signals, i.e. calcium release from SR following sarcolemma depolarization, can be modified in skeletal 
muscle fibers made hypertrophic by chronic clembuterol administration, ${ }^{72,73}$ and by myostatin ablation. ${ }^{60}$

The release of calcium from SR is triggered by action potential generated at neuro-muscular junction (NMJ) and sensed by the dihydropyridine receptor (DHPR). This brings us to the chain of events which form the basis of the neural motor control and that is profoundly affected by all types of training. Cortical motor neurons and spinal motor neurons (see for reviews, Folland and Williams,${ }^{74}$ Mason et al. ${ }^{19}$ ) and even the NMJ and its efficiency, ${ }^{75}$ respond to training. Neural adaptations are essentially changes in coordination and learning how to improve recruitment and activation with appropriate firing rates of the muscles involved in a specific strength task.

Last but not least, the changes in muscle architecture, and in particular the increase in pennation angle with hypertrophy first reported by Gollnick et al $(1981)^{76}$ can alter the relation between muscle size and contractile performance. ${ }^{77}$ The change in tendon properties, ${ }^{78}$ can modify the internal shortening during isometric contraction and possibly alter the segment of the lengthtension curve where sarcomeres operate. ${ }^{79}$

In summary, the above list, although not exhaustive, shows that there is a wealth of factors, which can produce the dissociation of gain in strength and gain in mass in skeletal muscles after overload in relation to resistance training as well as after hormone administration or genetic manipulations.

\section{Conclusions and perspectives}

The presently available data support the view that the relation between increase in muscle size and in muscle contractile performance is far from being simple and straightforward. Different types of muscle adaptations converge under the model of hypertrophy, defined as an increase of muscle mass with a constant number of fibers. Specific components of the muscles, such as myofibrils and sarcoplasm with organelles inside muscle fibers and ECM around them, react differently to specific stimuli and trigger specific responses, which may involve the adaptation of the contractile machinery.

There is not only one type of hypertrophy and the study of the relation between increase in mass and increase in force helps to identify each specific type of hypertrophy and to select suitable means (training protocols, nutrition, pharmacological interventions) to achieve it. The progress of the knowledge on different types of hypertrophy and how to obtain them is relevant not only for basic myology. The relation between increase of mass and improvement of performance is relevant to the sport field and also for rehabilitation after conditions, which have caused atrophy or for prevention of sarcopenia and cachexia.

\section{List of acronyms}

BFR - Blood Flow Restricted

BFRRE - Blood Flow Restricted Resistance Exercise
CSA - cross-sectional area

CT - computed tomography

DEXA - dual-energy x-ray absorptiometry

DHPR -. dihydropyridine receptor

ECM - extracellular matrix

FSR - fractional synthesis rate

LA - levator ani

MRI - magnetic resonance imaging

MVC - maximal voluntary contraction

NMJ -. neuro-muscular jucion

PCSA - physiological cross-sectional area

TEM - transmission electron microscopy

\section{Authors contributions}

CR and SS equally contributed and approved the final typescript.

\section{Acknowledgments}

Funding This work has been co-funded by the Slovenian Research Agency research programme P5-0381 Kinesiology for Quality of Life and by ASI, MARS-PRE Project, n. DC-VUM-2017-006.

\section{Conflict of Interest}

The authors have no conflicts to disclose.

\section{Ethical Publication Statement}

We confirm that we have read the Journal's position on issues involved in ethical publication and affirm that this report is consistent with those guidelines.

\section{Corresponding Author}

Carlo Reggiani, Department of Biomedical Sciences, University of Padova, Via Ugo Bassi, 58/B 35131, Padova, Italy. ORCID iD: 0000-0001-8080-361X

Email: carlo.reggiani@unipd.it

E-mail and ORCID iD of the co-author

Stefano Schiaffino: stefano.schiaffino@unipd.it ORCID iD: 0000-0002-5607-6421

\section{References}

1. Gentil P, de Lira CAB, Paoli A, et al. Nutrition, pharmacological and training strategies adopted by six bodybuilders: case report and critical review. Eur J Transl Myol 2017;27(1):6247. Published 2017 Mar 24. doi:10.4081/ejtm.2017.6247

2. Ikegawa S, Funato K, Tsunoda N, et al. Muscle force per cross-sectional area is inversely related with pennation angle in strength trained athletes. J Strength Cond Res 2008;22:128-31. doi:10.1519/ JSC.0b013e $31815 \mathrm{f} 2 \mathrm{fd} 3$

3. Fry AC. The role of resistance exercise intensity on muscle fibre adaptations. Sports Med 2004;34:66379. doi: 10.2165/00007256-200434100-00004

4. Rasch PJ. The problem of muscle hypertrophy: a review. J Am Osteopath Assoc 1955;54:525-8. 
5. Moritani T, deVries HA. Neural factors versus hypertrophy in the time course of muscle strength gain. Am J Phys Med 1979;58:115-30.

6. Buckner SL, Dankel SJ, Mattocks KT, et al. The problem of muscle hypertrophy: Revisited. Muscle Nerve 2016;54:1012-4. doi:10.1002/mus.25420

7. Loenneke JP, Buckner SL, Dankel SJ, et al. Exercise-Induced Changes in Muscle Size do not Contribute to Exercise-Induced Changes in Muscle Strength. Sports Med 2019;49:987-91. https://doi.org/10.1007/s40279-019-01106-9

8. Loenneke JP, Dankel SJ, Bell ZW, et al. Is muscle growth a mechanism for increasing strength? Med Hypotheses 2019;125:51-6. doi:10.1016/j.mehy. 2019.02.030

9. Roberts MD, Haun CT, Vann CG, et al. Sarcoplasmic Hypertrophy in Skeletal Muscle: A Scientific 'Unicorn' or Resistance Training Adaptation? Front Physiol 2020;11:816. doi: 10. 3389/fphys.2020.00816

10. Tesch PA. Training for bodybuilding. In: Komi PV, editor. Strength and power in sport. 2nd ed. London: Blackwell Scientific, 1992; n.d. p. 370-80.

11 Gentil P, Del Vecchio FB, Paoli A, et al. Isokinetic Dynamometry and 1RM Tests Produce Conflicting Results for Assessing Alterations in Muscle Strength. J Hum Kinet 2017;56:19-27. Doi: 10.1515/hukin-2017-0019

12. Buckner SL, Jessee MB, Mattocks KT, et al. Determining Strength: A Case for Multiple Methods of Measurement. Sports Med 2017;47:193-5. doi:10.1007/s40279-016-0580-3

13. Ahtiainen JP, Walker S, Peltonen $\mathrm{H}$, et al. Heterogeneity in resistance training-induced muscle strength and mass responses in men and women of different ages. Age (Dordr) 2016;38:10. doi: 10.1007/s11357-015-9870-1

14. Erskine RM, Fletcher G, Folland JP. The contribution of muscle hypertrophy to strength changes following resistance training. Eur J Appl Physiol 2014;114:1239-49. Doi: 10.1007/s00421014-2855-4

15. Maughan RJ, Watson JS, Weir J. Strength and cross-sectional area of human skeletal muscle. J Physiol (Lond) 1983;338:37-49. doi: 10.1113/ jphysiol.1983.sp014658

16. Alway SE, Stray-Gundersen J, Grumbt WH, et al. Muscle cross-sectional area and torque in resistance-trained subjects. Eur J Appl Physiol Occup Physiol 1990;60:86-90. doi: 10.1007/ BF00846026

17. Schoenfeld BJ, Contreras B, Krieger J, et al. Resistance Training Volume Enhances Muscle Hypertrophy but Not Strength in Trained Men. Med Sci Sports Exerc 2019;51:94-103. doi: 10.1249/ MSS.0000000000001764

18. Barbalho M, Coswig VS, Steele J, et al. Evidence for an Upper Threshold for Resistance Training
Volume in Trained Women. Med Sci Sports Exerc 2019;51:515-22. doi: 10.1249/MSS.000000000000 1818

19. Mason J, Frazer AK, Pearce AJ, et al. Determining the early corticospinal-motoneuronal responses to strength training: a systematic review and metaanalysis. Rev Neurosci 2019;30:463-76. doi: 10.1515/revneuro-2018-0054

20. Seynnes OR, Kamandulis S, Kairaitis R, et al. Effect of androgenic-anabolic steroids and heavy strength training on patellar tendon morphological and mechanical properties. J Appl Physiol 2013;115:84-9. doi: 10.1152/japplphysiol.01417. 2012

21. Widrick JJ, Stelzer JE, Shoepe TC, et al. Functional properties of human muscle fibers after short-term resistance exercise training. Am J Physiol Regul Integr Comp Physiol 2002;283:R408-416. doi: 10. 1152/ajpregu.00120.2002

22. Pansarasa O, Rinaldi C, Parente V, et al. Resistance training of long duration modulates force and unloaded shortening velocity of single muscle fibres of young women. J Electromyogr Kinesiol 2009;19:e290-300. doi: 10.1016/j.jelekin.2008.07. 007

23. Shoepe TC, Stelzer JE, Garner DP, et al. Functional adaptability of muscle fibers to long-term resistance exercise. Med Sci Sports Exerc 2003;35:944-51. doi: 10.1249/01.MSS.0000069756.17841.9E

24. D'Antona G, Lanfranconi F, Pellegrino MA, et al. Skeletal muscle hypertrophy and structure and function of skeletal muscle fibres in male body builders. J Physiol (Lond) 2006;570:611-27. doi: 10.1113/jphysiol.2005.101642

25. Meijer JP, Jaspers RT, Rittweger J, et al. Single muscle fibre contractile properties differ between body-builders, power athletes and control subjects. Exp Physiol 2015;100:1331-41. doi: 10.1113/EP 085267

26. Claassen H, Gerber C, Hoppeler H, et al. Muscle filament spacing and short-term heavy-resistance exercise in humans. $\mathbf{J}$ Physiol (Lond) 1989;409:491-5. doi: 10.1113/jphysiol.1989.sp 017509

27. MacDougall JD, Sale DG, Elder GCB, et al. Muscle ultrastructural characteristics of elite powerlifters and bodybuilders. Europ J Appl Physiol 1982;48:117-26. doi: 10.1007/BF00421171

28. Toth MJ, Miller MS, VanBuren P, et al. Resistance training alters skeletal muscle structure and function in human heart failure: effects at the tissue, cellular and molecular levels. J Physiol (Lond) 2012;590:1243-59. doi: 10.1113/jphysiol.2011. 219659

29. MacDougall JD, Ward GR, Sale DG, et al. Biochemical adaptation of human skeletal muscle to heavy resistance training and immobilization. J 
Appl Physiol Respir Environ Exerc Physiol 1977;43:700-3. doi: 10.1152/jappl.1977.43.4.700

30. Haun CT, Vann CG, Osburn SC, et al. Muscle fiber hypertrophy in response to 6 weeks of high-volume resistance training in trained young men is largely attributed to sarcoplasmic hypertrophy. PLoS ONE 2019;14:e0215267. doi: 10.1371/journal.pone.0215 267

31. Brook MS, Wilkinson DJ, Mitchell WK, et al. Skeletal muscle hypertrophy adaptations predominate in the early stages of resistance exercise training, matching deuterium oxidederived measures of muscle protein synthesis and mechanistic target of rapamycin complex 1 signaling. The FASEB Journal 2015;29:4485-96. doi: 10.1096/fj.15-273755

32. Ato S, Tsushima D, Isono Y, et al. The Effect of Changing the Contraction Mode During Resistance Training on mTORC1 Signaling and Muscle Protein Synthesis. Front Physiol 2019;10:406. doi: 10.3389/fphys.2019.00406

33. Wilkinson DJ, Franchi MV, Brook MS, et al. A validation of the application of $\mathrm{D}(2) \mathrm{O}$ stable isotope tracer techniques for monitoring day-to-day changes in muscle protein subfraction synthesis in humans. Am J Physiol Endocrinol Metab 2014;306:E571-579. doi: 10.1152/ajpendo.00650. 2013

34. Vissing K, Groennebaek T, Wernbom M, et al. Myocellular Adaptations to Low-Load Blood Flow Restricted Resistance Training. Exercise and Sport Sciences Reviews 2020; Publish Ahead of Print: doi: 10.1249/JES.0000000000000231

35. Nielsen JL, Aagaard P, Bech RD, et al. Proliferation of myogenic stem cells in human skeletal muscle in response to low-load resistance training with blood flow restriction. J Physiol 2012;590:4351-61. doi: 10.1113/jphysiol.2012.237008

36. Fitts RH, Peters JR, Dillon EL, et al. Weekly versus monthly testosterone administration on fast and slow skeletal muscle fibers in older adult males. J Clin Endocrinol Metab 2015;100:E223-231. doi: 10.1210/jc.2014-2759

37. Lamboley CR, Xu H, Dutka TL, et al. Effect of androgen deprivation therapy on the contractile properties of type I and type II skeletal muscle fibres in men with non-metastatic prostate cancer. Clin Exp Pharmacol Physiol 2018;45:146-54. https://doi.org/10.1111/1440-1681.12873

38. Roy RR, Wilson R, Edgerton VR. Architectural and mechanical properties of the rat adductor longus: response to weight-lifting training. Anat Rec 1997;247:170-8. doi:1002/(SICI)10970185(199702)247:2<170::AID-AR3>3.0.CO;2-1

39. Goldberg AL. Work-induced growth of skeletal muscle in normal and hypophysectomized rats. Am J Physiol 1967;213:1193-8. doi: 10.1152/ajplega cy.1967.213.5.1193
40. Hubbard RW, Ianuzzo CD, Mathew WT, et al. Compensatory adaptations of skeletal muscle composition to a long-term functional overload. Growth 1975;39:85-93.

41. Kandarian SC, White TP. Mechanical deficit persists during long-term muscle hypertrophy. J Appl Physiol 1990;69:861-7. doi: 10.1152/jappl. 1990.69.3.861

42. Pérez-Schindler J, Summermatter S, Santos G, et al. The transcriptional coactivator PGC- $1 \alpha$ is dispensable for chronic overload-induced skeletal muscle hypertrophy and metabolic remodeling. Proc Natl Acad Sci USA 2013;110:20314-9. doi: 10.1073/pnas. 1312039110

43. Mendias CL, Schwartz AJ, Grekin JA, et al. Changes in muscle fiber contractility and extracellular matrix production during skeletal muscle hypertrophy. J Appl Physiol 2017;122:571-9. doi: 10.1152/japplphysiol.00719.2016

44. Antonio-Santos J, Ferreira DJS, Gomes Costa GL, et al. Resistance Training Alters the Proportion of Skeletal Muscle Fibers but Not Brain Neurotrophic Factors in Young Adult Rats. J Strength Cond Res 2016;30:3531-8. doi: 10.1519/JSC.000000000000 1449

45. Duncan ND, Williams DA, Lynch GS. Adaptations in rat skeletal muscle following long-term resistance exercise training. Eur J Appl Physiol Occup Physiol 1998;77:372-8. doi: 10.1007/ s004210050347

46. Cui D, Drake JC, Wilson RJ, et al. A novel voluntary weightlifting model in mice promotes muscle adaptation and insulin sensitivity with simultaneous enhancement of autophagy and mTOR pathway. FASEB J 2020;34:7330-44. doi: 10.1096/fj.201903055R

47. Dungan CM, Murach KA, Frick KK, et al. Elevated myonuclear density during skeletal muscle hypertrophy in response to training is reversed during detraining. Am J Physiol, Cell Physiol 2019;316:C649-54. doi: 10.1152/ajpcell.00050. 2019

48. Seldeen KL, Lasky G, Leiker MM, et al. High Intensity Interval Training Improves Physical Performance and Frailty in Aged Mice. J Gerontol A Biol Sci Med Sci 2018;73:429-37. doi:1093/ gerona/glx 120

49. Goh Q, Song T, Petrany MJ, et al. Myonuclear accretion is a determinant of exercise-induced remodeling in skeletal muscle. Elife 2019;8:e44876. doi:10.7554/eLife.44876

50. Tobin C, Joubert Y. Testosterone-induced development of the rat levator ani muscle. Dev Biol 1991;146:131-8. doi: 10.1016/0012-1606(91)90 453-a

51. Ye F, McCoy SC, Ross HH, et al. Transcriptional regulation of myotrophic actions by testosterone and trenbolone on androgen-responsive muscle. 
Steroids 2014;87:59-66. doi: 10.1016/j.steroids. 2014.05.024

52. Ustünel I, Akkoyunlu G, Demir R. The effect of testosterone on gastrocnemius muscle fibres in growing and adult male and female rats: a histochemical, morphometric and ultrastructural study. Anat Histol Embryol 2003;32:70-9. doi: 10.1046/j.1439-0264.2003.00441.x

53. Axell A-M, MacLean HE, Plant DR, et al. Continuous testosterone administration prevents skeletal muscle atrophy and enhances resistance to fatigue in orchidectomized male mice. Am J Physiol Endocrinol Metab 2006;291:E506-516. doi:10.1152/ajpendo.00058.2006

54. Zeman RJ, Ludemann R, Easton TG, et al. Slow to fast alterations in skeletal muscle fibers caused by clenbuterol, a beta 2-receptor agonist. Am J Physiol 1988;254:E726-732. doi:10.1152/ajpendo.1988. 254.6.E726

55. Lynch GS, Hayes A, Campbell SP, et al. Effects of beta 2-agonist administration and exercise on contractile activation of skeletal muscle fibers. J Appl Physiol 1996;81:1610-8. doi: 10.1152/jappl. 1996.81.4.1610

56. Polla B, Cappelli V, Morello F, et al. Effects of the beta(2)-agonist clenbuterol on respiratory and limb muscles of weaning rats. Am J Physiol Regul Integr Comp Physiol 2001;280:R862-869. doi: 10.1152/ ajpregu.2001.280.3.R862

57. Kim J, Grotegut CA, Wisler JW, et al. $\beta$-arrestin 1 regulates $\beta 2$-adrenergic receptor-mediated skeletal muscle hypertrophy and contractility. Skelet Muscle 2018;8:39. doi: 10.1186/s13395-018-0184-8

58. Kambadur R, Sharma M, Smith TP, et al. Mutations in myostatin (GDF8) in double-muscled Belgian Blue and Piedmontese cattle. Genome Res 1997;7:910-6. doi: 10.1101/gr.7.9.910

59. Amthor H, Macharia R, Navarrete R, et al. Lack of myostatin results in excessive muscle growth but impaired force generation. PNAS 2007;104:183540. doi: 10.1073/pnas.0604893104

60. Bodnár D, Geyer N, Ruzsnavszky O, et al. Hypermuscular mice with mutation in the myostatin gene display altered calcium signalling. J Physiol (Lond) 2014;592:1353-65. doi: 10.1113/jphysiol. 2013.261958

61. Blaauw B, Canato M, Agatea L, et al. Inducible activation of Akt increases skeletal muscle mass and force without satellite cell activation. FASEB J 2009;23:3896-905. doi: 10.1096/fj.09-131870

62. Musarò A, McCullagh K, Paul A, et al. Localized Igf-1 transgene expression sustains hypertrophy and regeneration in senescent skeletal muscle. Nat Genet 2001;27:195-200. doi: 10.1038/84839

63. Ascenzi F, Barberi L, Dobrowolny G, et al. Effects of IGF-1 isoforms on muscle growth and sarcopenia. Aging Cell 2019;18:e12954. doi: 10.1111/acel.12954
64. Gonzalez E, Messi ML, Zheng Z, et al. Insulin-like growth factor-1 prevents age-related decrease in specific force and intracellular $\mathrm{Ca} 2+$ in single intact muscle fibres from transgenic mice. J Physiol (Lond) 2003;552:833-44. doi: 10.1113/jphysiol. 2003.048165

65. Colombini B, Benelli $G$, Nocella $M$, et al. Mechanical properties of intact single fibres from wild-type and MLC/mIgf-1 transgenic mouse muscle. J Muscle Res Cell Motil 2009;30:199-207. doi: 10.1007/s10974-009-9187-8

66 Percario V, Boncompagni S, Protasi F, et al. Mechanical parameters of the molecular motor myosin II determined in permeabilised fibres from slow and fast skeletal muscles of the rabbit. J Physiol (Lond) 2018;596:1243-57. doi:10.1113/JP 275404

67. Schiaffino S, Reggiani C. Fiber types in mammalian skeletal muscles. Physiol Rev 2011;91:1447-531. doi: 10.1152/physrev.00031.2010

68. Canepari M, Rossi R, Pellegrino MA, et al. Effects of resistance training on myosin function studied by the in vitro motility assay in young and older men. J Appl Physiol 2005;98:2390-5. doi: 10.1152/ japplphysiol.01103.2004

69. Marcucci L, Washio T, Yanagida T. Titin-mediated thick filament activation, through a mechanosensing mechanism, introduces sarcomere-length dependencies in mathematical models of rat trabecula and whole ventricle. Scientific Reports 2017;7:5546. doi: 10.1038/s 41598-017-05999-2

70. Irving M. Regulation of Contraction by the Thick Filaments in Skeletal Muscle. Biophys J 2017;113:2579-94. doi: 10.1016/j.bpj.2017.09.037

71. Lehman W. Thin Filament Structure and the Steric Blocking Model. Compr Physiol 2016;6:1043-69. doi: 10.1002/cphy.c150030

72. Sirvent P, Douillard A, Galbes O, et al. Effects of chronic administration of clenbuterol on contractile properties and calcium homeostasis in rat extensor digitorum longus muscle. PLoS ONE 2014;9:e100281. doi: 10.1371/journal.pone.0100281

73. Py G, Ramonatxo C, Sirvent P, et al. Chronic clenbuterol treatment compromises force production without directly altering skeletal muscle contractile machinery. J Physiol (Lond) 2015;593:2071-84. Doi: 10.1113/jphysiol.2014. 287060

74. Folland JP, Williams AG. The adaptations to strength training: morphological and neurological contributions to increased strength. Sports Med 2007;37:145-68. doi: 10.2165/00007256-2007370 20-00004

75. Deschenes MR, Tufts HL, Oh J, et al. Effects of exercise training on neuromuscular junctions and their active zones in young and aged muscles. 
Neurobiol Aging 2020;95:1-8. doi: 10.1016/j.neuro biolaging.2020.07.001

76. Gollnick PD, Timson BF, Moore RL, et al. Muscular enlargement and number of fibers in skeletal muscles of rats. Journal of Applied Physiology 1981;50:936-43. doi: 10.1152/jappl. 1981.50.5.936

77. Franchi MV, Atherton PJ, Reeves ND, et al. Architectural, functional and molecular responses to concentric and eccentric loading in human skeletal muscle. Acta Physiol (Oxf) 2014;210:64254. doi: 10.1111/apha.12225
78. Reeves ND, Narici MV, Maganaris CN. Myotendinous plasticity to ageing and resistance exercise in humans. Exp Physiol 2006;91:483-98. doi 10.1113/expphysiol.2005.032896

79. Reeves ND, Narici MV, Maganaris CN. In vivo human muscle structure and function: adaptations to resistance training in old age. Exp Physiol 2004;89:675-89. doi: 10.1113/expphysiol.2004. 027797

Submitted: August 17, 2020 Accepted for publication: August 23, 2020 